\title{
BMJ Open Web-based MINDfulness and Skills- based distress reduction in cancer (MINDS): study protocol for a multicentre observational healthcare study
}

\author{
Alexander Bäuerle (10 , ,,2 Martin Teufel, ${ }^{1,2}$ Caterina Schug, ${ }^{3}$ Eva-Maria Skoda, ${ }^{1,2}$ \\ Mingo Beckmann, ${ }^{1,2}$ Norbert Schäffeler, ${ }^{4,5}$ Florian Junne, ${ }^{4,5}$ Yesim Erim, ${ }^{3}$ \\ Stephan Zipfel, ${ }^{4,5}$ Johanna Graf ${ }^{4,5}$
}

To cite: Bäuerle A, Teufel M, Schug C, et al. Web-based MINDfulness and Skills-based distress reduction in cancer (MINDS): study protocol for a multicentre observational healthcare study. BMJ Open 2020;10:e036466. doi:10.1136/ bmjopen-2019-036466

- Prepublication history and additional material for this paper are available online. To view these files, please visit the journal online (http://dx.doi. org/10.1136/bmjopen-2019036466).

Received 16 December 2019 Revised 30 March 2020 Accepted 13 July 2020

Check for updates

(C) Author(s) (or their employer(s)) 2020. Re-use permitted under CC BY-NC. No commercial re-use. See rights and permissions. Published by BMJ.

For numbered affiliations see end of article.

Correspondence to

Alexander Bäuerle;

alexander.baeuerle@uni-due.de

\section{ABSTRACT}

Introduction Although a high percentage of patients with cancer experience severe psychological distress, few of them receive psycho-oncological care, largely due to barriers on the side of patients and healthcare providers that pose great challenges to delivering such care. In response, low-threshold, self-guided eHealth interventions can enable patients with cancer to deal independently and effectively with disease-related challenges and distress. Mindfulness and Skills-Based Distress Reduction in Oncology Training, nicknamed Make It Training, is one such innovative, self-guided eHealth intervention. In our study, we propose to assess different characteristics of such patients in order to define target populations for Make It Training, evaluate the intervention in terms of its usability, feasibility and sustainability and gather longitudinal data concerning the intervention's efficacy.

Methods and analysis Self-guided and web-based Make It Training consists of eight 30 min modules involving the use of techniques of mindfulness therapy, cognitive-behavioural therapy and acceptance and commitment therapy to be completed in a 4-month period. In our observational study, adult patients with cancer who possess adequate German language skills and provide their informed consent will be recruited at Essen, Erlangen and Tübingen University Hospitals at outpatient oncological institutions and via online channels. Patients will undergo a baseline online assessment (T0), an assessment directly after completing the intervention (T1) and assessments 3 and 6 months later (T2 and T3, respectively). With the results of those assessments, we will perform descriptive analyses of their sociodemographic and medical data, compare means and conduct regression analyses.

Ethics and dissemination The Ethics Committees of the University Hospitals Essen, Erlangen and Tübingen have approved the study (19-8643-B0, 27_19 B, 293/2018B01). Results will be published in peer-reviewed journals and conference presentations.

Trial registration number DRKS00017119.
Strengths and limitations of this study

- The web-based intervention was developed using evidence, theory and person-based approaches.

- The study will evaluate the intervention in terms of acceptance, usability, feasibility and sustainability and collect longitudinal data on the effectiveness of the web-based approach.

- Provision of the web-based training for a large number of patients with cancer independent of the tumour entity.

No randomised controlled study design.

\section{INTRODUCTION}

Despite considerable improvements in the treatment of different tumour entities, every second patient with cancer suffers from acute psychological distress. ${ }^{1}$ In fact, a third of all patients with cancer involving major tumour entities will meet the criteria for at least one mental health disorder at 4-week prevalence. ${ }^{2}$ Over a lifetime, however, the prevalence of any mental disorder in patients with cancer rises to more than $56 \% .^{3}$ Psychological distress in patients with cancer is often associated with changed roles in their professional and family lives, financial problems and reduced quality of life (QoL) $){ }^{4-6}$ and high distress can even reduce compliance with treatment. ${ }^{78}$ As a partial antidote, psycho-oncological interventions can significantly reduce the psychological distress and improve the QoL of patients with cancer. $^{9}{ }^{10}$ Although many patients with cancer demonstrate a great need for adequate psycho-oncological care in order to overcome disease-related distress, depression and anxiety, ${ }^{11}{ }^{12}$ few of them receive such care. ${ }^{13}$ In response, it is crucial for healthcare institutions to offer patients with cancer 
usable, sustainable and accessible interventions, such as eHealth interventions involving psycho-oncological treatment approaches for routine clinical care. ${ }^{14}$

\section{Cognitive-behavioural therapy and mindfulness in psycho- oncological interventions}

Various studies have revealed that cognitive-behavioural therapy (CBT) and mindfulness-based stress reduction (MBSR) significantly reduce distress, depression and anxiety in patients with cancer. ${ }^{9} 1516$ Such approaches can also increase their QoL and reduce the treatmentrelated side effects that patients with cancer may experience. ${ }^{7817}$ To date, the effect sizes of psycho-oncological interventions based on CBT and MBSR have been small to medium in randomised controlled trials (RCT). ${ }^{18}$

\section{Psycho-oncological eHealth intervention}

Given the increasing influence of internet use in daily life, web-based psycho-oncological care has the potential to reach high volumes of patients and to overcome barriers to healthcare provision on the side of patients and providers (eg, rural areas, stigma, privacy, high costs and long wait times) ${ }^{19}{ }^{20}$ In particular, eHealth interventions can be efficient approaches to reaching younger patients who report more unmet needs, are less likely to access psycho-oncological help but are generally more open to modern and web-based approaches. ${ }^{21}$ At the same time, easy integration of psycho-oncological care through eHealth approaches into patients' lives prevents an additional workload for this vulnerable group. ${ }^{21}$ Compared with the effects of face-to-face CBT interventions in several mental and somatic disorders, online CBT interventions lead to comparable efficacy outcomes. ${ }^{22} 23$ Other promising results suggest that online psycho-oncological interventions can improve the psychosocial and physical symptoms of patients with cancer. ${ }^{24-26}$ There are more approaches to address the different needs of patients with cancer. ${ }^{27}{ }^{28}$ Nevertheless, due to the mixed findings and small sample sizes of studies on such interventions to date, high-quality research with larger, more diverse samples remains necessary. ${ }^{29} 30$

\section{Objectives and research questions}

To overcome the current gap in the provision of psychooncological care for patients with cancer, we have developed an innovative eHealth intervention named Make It Training. Highly time and cost efficient, Make It Training provides a novel way to support patients with cancer regardless of their physical conditions or location by improving their social and professional functioning and engagement. The chief objective of our study is to evaluate the acceptability, usability, feasibility and sustainability of Make It Training. In particular, we seek to identify the primary characteristics of patients with cancer in order to determine target populations for the intervention, namely by evaluating:

1. Acceptance and adherence to Make It Training.

2. Dropout in and the usability of Make It Training.

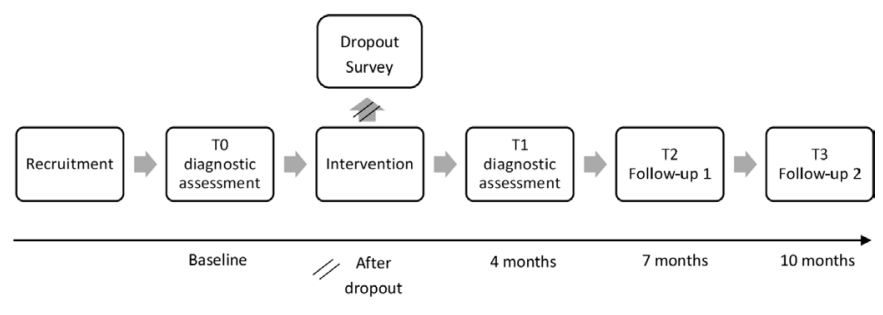

Figure 1 Overall study design.

Our secondary objectives refer to initial indications of the efficacy of Make It Training in order to identify patient characteristics that might determine potential benefits for patients with cancer. To that end, we developed two research questions:

1. Does Make It Training lead to increased self-efficacy, QoL and mindfulness among patients with cancer?

2. Does Make It Training lead to reduced distress, depression and anxiety among patients with cancer?

Because developing modern, issue-based interventions is important to improve patients' acceptance of eHealth interventions, ${ }^{22}$ the results of our study promise to afford broad knowledge applicable to identifying and addressing specific groups of patients with cancer who are open to and may benefit from eHealth interventions in psychooncological care.

\section{METHODS AND ANALYSIS \\ Study design}

Our multicentre observational healthcare study is designed to offer the web-based intervention of Make It Training to a large number of patients with cancer. The proposed procedure for the study appears in figure 1 . Once cancer diagnosis is determined from their medical records, participating patients will complete the online baseline assessment (T0) and receive their personal Make It Training usernames from the research team. Further assessments will be made directly after the completion of the intervention (T1) and at both 3 and 6 months later (T2 and T3, respectively). In the case of discontinuation (ie, no login for 6 weeks after the first uncompleted module), patients will be asked to complete a questionnaire addressing their reasons for not completing Make It Training. During the intervention, each patient will receive a notification every 2 weeks stating that a new module is accessible. To improve study adherence, patients who have been inactive for 4 weeks will receive an additional notification to motivate them to continue Make It Training. The intervention will be considered to be complete when at least five of eight modules have been conducted.

\section{Participant eligibility and recruitment}

Patients with cancer will be included regardless of their demographic or medical characteristics (eg, age and tumour entity), provided that they have a good command of the German language, internet access and basic computer skills, are at least 18 years of age and have 
given their informed consent. Patients will be recruited at University Hospitals Essen, Erlangen and Tübingen, at outpatient cancer institutions (eg, established physicians), with the distribution of flyers and from the publicly accessible website for Make It Training. ${ }^{31}$ Patients will be recruited from self-help and other groups on social media. At all three university hospitals, we will contact all patients with cancer who have indicated interest in participating in studies for patients with cancer and send them informational material about our study via email. Their informed consent will be given with their online confirmation of the study conditions in advance of the first assessment.

\section{eHealth intervention: Make It Training}

Make It Training is a self-guided, interactive and webbased intervention that patients can use on their own personal computers, smartphones or tablets. Make It Training is based on established, effective methods of CBT, mindfulness therapy and acceptance and commitment therapy. ${ }^{9} 151632$ By combining both skills training and practices of mindfulness, Make It Training can address the different needs of psychologically burdened patients with cancer. ${ }^{33}$ A previous pilot study with $\mathrm{n}=35$ has demonstrated the good acceptance and contentment rates of an exemplary module of the Make It Training. ${ }^{34}$

The eight modules of Make It Training contain different media-tutorial videos, audio, individual skills boxes and patient-centred, interactive exercises-to enhance the acquisition of knowledge about specific disease-related topics (eg, emotion management, resources, stress management and self-compassion). All guided mindfulness exercises are available as audio files and can be downloaded onto the patients' devices. Table 1 gives an overview of the contents of the individual modules of Make It Training translated into English. ${ }^{34}$ Make It Training consists of eight 30 min modules offered at the rate of one module every 2 weeks for 4 months. In addition to the eight modules, mindfulness exercises to be performed at home should be integrated into patients' daily routines. The interval of 2 weeks per module was

Table 1 Overview of the topics, contents and exercises of Make It Training

\begin{tabular}{lllll}
\hline & Topic & Psychoeducation & Skills & Mindfulness \\
\hline $\begin{array}{l}\text { 1. Module } \\
\text { Introduction }\end{array}$ & $\begin{array}{l}\text { The idea of the skills and } \\
\text { mindfulness training and } \\
\text { skills }\end{array}$ & $\begin{array}{l}\text { No psychoeducation } \\
\text { because of introduction }\end{array}$ & $\begin{array}{l}\text { No skills training } \\
\text { because of intense } \\
\text { mindfulness exercise }\end{array}$ & Mindful drinking \\
2. Module & Accepting and dealing & $\begin{array}{l}\text { The presence of cancer- } \\
\text { related emotions such as }\end{array}$ & - Exercise: Giving \\
Emotions & with emotions space & Mindful breathing \\
& & anxiety, sadness and anger & Distance strategies \\
& & Acceptance cards
\end{tabular}

$\begin{array}{llll}\begin{array}{l}\text { 3. Module } \\ \text { Experiencing }\end{array} & \begin{array}{l}\text { Experiencing one's } \\ \text { environment without } \\ \text { judging it }\end{array} & \begin{array}{l}\text { No psychoeducation } \\ \text { because of intense } \\ \text { mindfulness exercise }\end{array} & \begin{array}{l}\text { No skills training } \\ \text { because of intense } \\ \text { mindfulness exercise }\end{array} \\ \begin{array}{l}\text { 4. Module } \\ \text { Sources of strength }\end{array} & \begin{array}{l}\text { Discovering and } \\ \text { consciously using } \\ \text { personal sources of } \\ \text { strength }\end{array} & \begin{array}{l}\text { The emergence of vicious } \\ \text { mental cycles and the } \\ \text { meaning of individual }\end{array} & \begin{array}{l}\text { Exercise: Exiting } \\ \text { the vicious cycle } \\ \text { by using personal } \\ \text { sources of strength }\end{array} \\ & & \text { sources of } \\ & & \text { Actiongth cards }\end{array}$

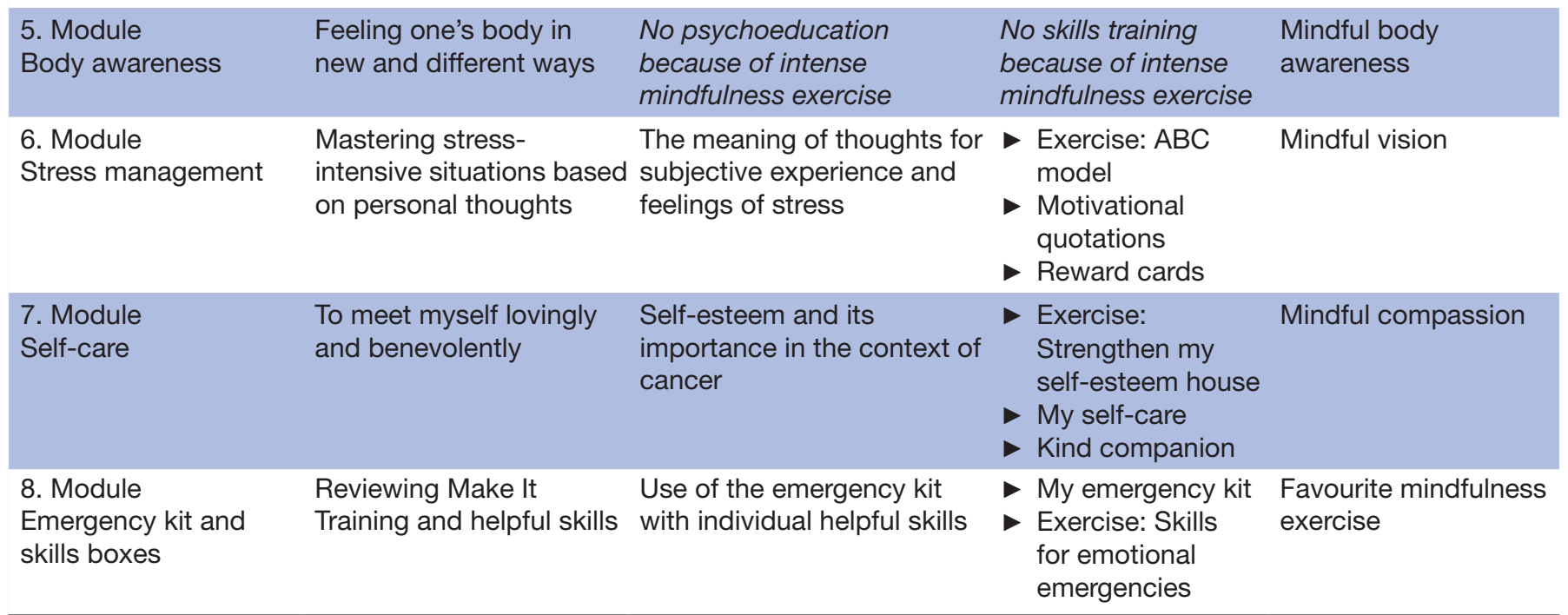




\section{Assessment time points}

\begin{tabular}{|c|c|c|c|c|}
\hline Measures & T0: baseline & T1: post intervention & $\begin{array}{l}\text { T2 and T3: follow-up } \\
\text { at } 3 \text { and } 6 \text { months }\end{array}$ & Dropout assessment \\
\hline $\begin{array}{l}\text { Demographic and medical } \\
\text { characteristics }\end{array}$ & $x$ & & & \\
\hline \multicolumn{5}{|l|}{ Evaluation of Make It Training } \\
\hline SUS & & $x$ & & $x$ \\
\hline APOI & & $x$ & & $x$ \\
\hline Self-generated evaluation items & & $x$ & & $x$ \\
\hline \multicolumn{5}{|l|}{ Secondary outcomes } \\
\hline DT & $x$ & $x$ & $x$ & $x$ \\
\hline PHQ-8 & $x$ & $x$ & $x$ & $x$ \\
\hline FMI & $x$ & $x$ & $x$ & $x$ \\
\hline PSQ-20 & $x$ & $x$ & $x$ & $x$ \\
\hline EQ-5D-3L & $x$ & $x$ & $x$ & $x$ \\
\hline FACT-G & $x$ & $x$ & $x$ & $x$ \\
\hline
\end{tabular}

See online supplementary appendix 1 for detailed information on demographic and medical characteristics.

APOI, Attitudes towards Psychological Online Interventions; CSQ-I, Client Satisfaction Questionnaire adapted to Internet-based interventions; DT, Distress Thermometer; EQ-5D-3L, European Quality of Life 5 Dimensions 3 Level Version; FACT-G, Functional Assessment of Cancer Therapy-General; ; FMI, Freiburg Mindfulness Inventory; GAD-7, Generalized Anxiety Disorder Scale-7; GSES, General Self-Efficacy Scale; PHQ-8, 8-item Patient Health Questionnaire Depression Scale; PSQ-20, Perceived Stress Questionnaire-20; SUS, System Usability Scale.

chosen to afford patients' sufficient time to integrate mindfulness exercises into their daily lives, which should improve their likelihood of continuing the exercises once the intervention has ended.

\section{Primary outcome measures}

The schedule of the different assessments, each lasting between 10 and $25 \mathrm{~min}$, is summarised in table 2. Demographic data (eg, gender, age, children, family status and employment status) and medical data (eg, tumour entity, treatment method, date of diagnosis and type of therapy) will be self-reported and collected via the online survey application Unipark before the patients start the intervention (T0). ${ }^{35}$

\section{Secondary outcome measures}

We will use the German version of the Distress Thermometer to assess patients' distress experienced in the past week. ${ }^{36}$ By contrast, depression and anxiety will be assessed with the German versions of the 8-item Patient Health Questionnaire Depression Scale and the Generalized Anxiety Disorder Scale-7, respectively. ${ }^{37} 38$ To gauge self-efficacy, we plan to use the German version of the General Self-Efficacy Scale (GSES), ${ }^{39}$ whereas to assess mindfulness, we propose to use the German version of the Freiburg Mindfulness Inventory. ${ }^{40}$ The German version of the Perceived Stress Questionnaire without the Joy subscale will be used to investigate and determine subjective perception, evaluation and the further processing of stressors. ${ }^{41}$ To gather relevant information about the current somatic state of patients, we will use the Physical Well-Being subscale of the German version of the Functional Assessment of Cancer Therapy-General. ${ }^{42}$ Last, to assess patients' quality of life, we plan to use the German version of the 5-item European Quality of Life 5 Dimensions 3 Level Version questionnaire. ${ }^{43}$

\section{Evaluation of Make It Training}

To evaluate the usability of and patients' satisfaction with Make It Training, we will use a modified German version of the 10-item System Usability Scale, ${ }^{44}$ the German version of the Client Satisfaction Questionnaire adapted to Internet-based interventions ${ }^{45}$ and self-generated items, all to gather detailed information about the strengths and weaknesses of Make It Training. For each item-based topic, patients will have the possibility to add their personal thoughts and ideas. The patients' acceptance of online psychological interventions in general and their possible changes in attitude will be collected with the Attitudes towards Psychological Online Interventions instrument. ${ }^{46}$ Reasons for not completing Make It Training will also be obtained, whereas data concerning adherence to the training (eg, last login and time needed 
for modules) will be collected via back-end functions of the Make It system.

\section{Sample size calculation and statistical analyses}

The sample size estimation will be done based on the Willems et al. ${ }^{26}$ in which an effect size of Cohen's $d=0.28$ was observed for GSES. ${ }^{39}$ Based on that effect size, we have calculated the need for a sample size of 103 patients using two-sided t-tests for dependent samples with type 1 error of 0.05 and a power of $80 \%$. Although a dropout rate of up to $50 \%$ for online interventions has been shown, ${ }^{47}$ we might expect an even higher rate of dropout given the physically vulnerable patient group that will form our sample and because the last measurement will occur 6 months after the intervention has ended. Assuming that some patients will not complete the first online assessment and thus not start Make It Training after recruitment, ${ }^{48}$ we plan to recruit 500 patients at T0. We will perform standard techniques of data preparation with tests of normality and homogeneity as well as conduct different descriptive analyses of sociodemographic and medical data. The primary statistical analyses will be an analysis of variance with repeated measurements, t-tests for dependent samples (ie, with two-sided $\mathrm{p}$ values and $95 \%$ CIs) and regression analyses to identify potential predictors of treatment outcomes. We will consider stage of disease, mindfulness experience and psychiatric comorbidities as potential covariates, include these in an additional regression model and perform moderator analyses considering these variables as moderator variables.

\section{Patient and public involvement}

Neither patients nor public were involved in the development of this study design, but as previously mentioned, patients were involved in developing the intervention content. ${ }^{33}$ Furthermore, we conducted a pilot study on the acceptance of an exemplary module of Make It Training. ${ }^{34}$ During the proposed study, we will also collect data on how Make It Training can be improved to subsequently improve it.

\section{Ethics and dissemination}

The Ethics Committees of the University Hospitals Essen, Erlangen and Tübingen have approved the study (19-8643-BO, 27_19 B, 293/2018BO1). Results will be disseminated in peer-reviewed journals and conference presentations. Key findings will also be published on the Make It Training website.

\section{Trial status}

Trial start date: 22 October 2019; currently recruiting $\left(\mathrm{n}_{\text {current }}=60\right.$ as of 16 December 2019).

\section{DISCUSSION}

In our study, we propose to evaluate whether specific groups of patients who differ in terms of sociodemographic data (eg, age, sex and family status) and medical data (eg, medical condition, time since cancer diagnosis and tumour entity) are generally open to using an innovative psycho-oncological eHealth intervention. Our evaluation is important in order to adapt modern interventions to the specific requirements and desires of such groups and to efficiently offer a low-threshold eHealth intervention. Our preliminary findings from a longitudinal perspective will suggest whether the self-guided, webbased Make It Training intervention is an efficient tool to support patients with cancer. In addition, we expect to be able to identify specific reasons for patient dropout that can help us to improve Make It Training in particular and to develop patient-centred eHealth interventions in general. A key topic of discussion for subsequent research will be whether a self-guided online intervention can be effectively used to support patients with cancer. Although various studies have highlighted the importance of therapeutic contact in online interventions, ${ }^{49}$ implementing such interventions in everyday healthcare necessarily faces numerous obstacles (eg, higher costs and mostly accessibility during research work only)..$^{50}$ In addition, evidence suggests that the efficacy of treatment between guided and self-guided online interventions for depression does not significantly differ. ${ }^{51}$ Make It Training attempts to overcome the mentioned barriers by offering a completely self-guided tool to support all patients with cancer anytime and anywhere. One expected limitation of the study is the non-existence of a control group to obtain sufficient data regarding the effectiveness of Make It Training. However, among its anticipated strengths is that we will offer Make It Training to all patients with cancer and gain valid information about the usability, feasibility and sustainability of web-based, self-guided psycho-oncological interventions. In a future study, we also plan to conduct a multicentre RCT to obtain valid information on the efficacy of Make It Training.

Although many patients with cancer suffer from acute psychological distress, psycho-oncological support is often absent, unavailable or non-existent. Make It Training is one eHealth approach to overcome barriers on the side of both the patient and the provider to offer patients with cancer a self-guided psycho-oncological intervention at any time and any place. Such interventions are time and cost efficient and afford broad availability in clinical routines for all patients at any stage of the disease, whether they are in acute care or have survived cancer. Make It Training in particular and self-guided eHealth interventions in general could be integrated in current healthcare systems to offer substantial benefits for patients and providers.

\section{Author affiliations}

${ }^{1}$ Clinic for Psychosomatic Medicine and Psychotherapy, LVR University-Hospital Essen, University of Duisburg-Essen, Essen, Germany

${ }^{2}$ Comprehensive Cancer Center Essen, University Hospital Essen, Essen, NordrheinWestfalen, Germany

${ }^{3}$ Department of Psychosomatic Medicine and Psychotherapy, University Hospital Erlangen, Friedrich-Alexander University Erlangen-Nürnberg, Erlangen, Bayern, Germany 
${ }^{4}$ Department of Psychosomatic Medicine and Psychotherapy, University Hospital Tübingen, Eberhard Karls University Tübingen, Tübingen, Baden-Württemberg, Germany

${ }^{5}$ Comprehensive Cancer Center Tübingen-Stuttgart, University Hospital Tübingen, Tübingen, Germany

Contributors $A B$ contributed to designing the study, administering the trial and preparing the manuscript. MT initiated the study and contributed to designing the study, developing the intervention, procuring funding and preparing the manuscript. $\mathrm{JG}$ contributed to designing the study, developing the intervention, administering the trial and preparing the manuscript. MB and EMS contributed to preparing the manuscript. NS and FJ contributed to developing the intervention and helping with the manuscript. CS contributed to administering the trial and preparing the manuscript. SZ contributed to designing the study, developing the intervention and procuring funding. YE contributed to designing the study, procuring funding and preparing the manuscript. All authors have read and approved the final manuscript.

Funding The Open Access Publication Fund of the University of Duisburg-Essen is supporting this open access publication.

Competing interests None declared.

Patient and public involvement Patients and/or the public were involved in the design, or conduct, or reporting, or dissemination plans of this research. Refer to the Methods section for further details.

Patient consent for publication Not required.

Ethics approval The Ethics Committees of the University Hospitals Essen (19-8643-B0), Erlangen (27_19 B) and Tübingen (293/2018B01) have approved the study.

Provenance and peer review Not commissioned; externally peer reviewed.

Open access This is an open access article distributed in accordance with the Creative Commons Attribution Non Commercial (CC BY-NC 4.0) license, which permits others to distribute, remix, adapt, build upon this work non-commercially, and license their derivative works on different terms, provided the original work is properly cited, appropriate credit is given, any changes made indicated, and the use is non-commercial. See: http://creativecommons.org/licenses/by-nc/4.0/.

ORCID iD

Alexander Bäuerle http://orcid.org/0000-0003-1488-8592

\section{REFERENCES}

1 Mehnert A, Hartung TJ, Friedrich M, et al. One in two cancer patients is significantly distressed: prevalence and indicators of distress. Psychooncology 2018;27:75-82.

2 Mehnert A, Brähler E, Faller H, et al. Four-week prevalence of mental disorders in patients with cancer across major tumor entities. J Clin Oncol 2014;32:3540-6.

3 Kuhnt S, Brähler E, Faller H, et al. Twelve-Month and lifetime prevalence of mental disorders in cancer patients. Psychother Psychosom 2016;85:289-96.

4 Singer S, Das-Munshi J, Brähler E. Prevalence of mental health conditions in cancer patients in acute care--a meta-analysis. Ann Oncol 2010;21:925-30.

5 Mitchell AJ, Chan M, Bhatti $\mathrm{H}$, et al. Prevalence of depression, anxiety, and adjustment disorder in oncological, haematological, and palliative-care settings: a meta-analysis of 94 Interview-Based studies. Lancet Oncol 2011;12:160-74.

6 Büttner M, König H-H, Löbner M, et al. Out-of-pocket-payments and the financial burden of 502 cancer patients of working age in Germany: results from a longitudinal study. Support Care Cancer 2019;27:2221-8.

7 Chambers SK, Meng X, Youl P, et al. A five-year prospective study of quality of life after colorectal cancer. Qual Life Res 2012;21:1551-64.

8 Park CL, Gaffey AE. Relationships between psychosocial factors and health behavior change in cancer survivors: an integrative review. Ann Behav Med 2007;34:115-34.

9 Faller H, Schuler M, Richard M, et al. Effects of psycho-oncologic interventions on emotional distress and quality of life in adult patients with cancer: systematic review and meta-analysis. J Clin Oncol 2013;31:782-93.

10 Cwikel JG, Behar LC. Social work with adult cancer patients: a vote-count review of intervention research. Soc Work Health Care 1999;29:39-67.
11 Faller $\mathrm{H}$, Weis $\mathrm{J}$, Koch $\mathrm{U}$, et al. Utilization of professional psychological care in a large German sample of cancer patients. Psychooncology 2017;26:537-43.

12 Schaeffeler N, Pfeiffer K, Ringwald J, et al. Assessing the need for psychooncological support: screening instruments in combination with patients' subjective evaluation may define psychooncological pathways. Psychooncology 2015;24:1784-91.

13 Zeissig SR, Singer S, Koch L, et al. Inanspruchnahme psychoonkologischer Versorgung Im Krankenhaus und in Krebsberatungsstellen durch Brust-, Darm- und Prostatakrebsüberlebende. Psychother Psych Med 2015;65:177-82.

14 Aaronson NK, Mattioli V, Minton O, et al. Beyond treatment Psychosocial and behavioural issues in cancer survivorship research and practice. EJC Supp/ 2014;12:54-64.

15 Zainal NZ, Booth S, Huppert FA. The efficacy of mindfulness-based stress reduction on mental health of breast cancer patients: a metaanalysis. Psychooncology 2013;22:1457-65.

16 Rush SE, Sharma M. Mindfulness-based stress reduction as a stress management intervention for cancer care: a systematic review. J Evid Based Complementary Altern Med 2017;22:348-60.

17 Wells N, Hepworth JT, Murphy BA, et al. Improving cancer pain management through patient and family education. J Pain Symptom Manage 2003;25:344-56.

18 Duijts SFA, Faber MM, Oldenburg HSA, et al. Effectiveness of behavioral techniques and physical exercise on psychosocial functioning and health-related quality of life in breast cancer patients and survivors--a meta-analysis. Psychooncology 2011;20:115-26.

19 Leykin Y, Thekdi SM, Shumay DM, et al. Internet interventions for improving psychological well-being in psycho-oncology: review and recommendations. Psychooncology 2012;21:1016-25.

20 Dilworth S, Higgins I, Parker V, et al. Patient and health professional's perceived barriers to the delivery of psychosocial care to adults with cancer: a systematic review. Psychooncology 2014;23:601-12.

21 Zebrack BJ, Block R, Hayes-Lattin B, et al. Psychosocial service use and unmet need among recently diagnosed adolescent and young adult cancer patients. Cancer 2013;119:201-14.

22 Andersson G. Internet-Delivered psychological treatments. Annu Rev Clin Psychol 2016;12:157-79.

23 Andersson G, Cuijpers P, Carlbring P, et al. Guided Internet-based vs. face-to-face cognitive behavior therapy for psychiatric and somatic disorders: a systematic review and meta-analysis. World Psychiatry 2014;13:288-95.

24 Bouma G, Admiraal JM, de Vries EGE, et al. Internet-based support programs to alleviate psychosocial and physical symptoms in cancer patients: a literature analysis. Crit Rev Oncol Hematol 2015;95:26-37.

25 Urech C, Grossert A, Alder J, et al. Web-Based stress management for newly diagnosed patients with cancer (stream): a randomized, Wait-List controlled intervention study. J Clin Oncol 2018;36:780-8.

26 Willems RA, Bolman CAW, Mesters I, et al. Short-term effectiveness of a web-based tailored intervention for cancer survivors on quality of life, anxiety, depression, and fatigue: randomized controlled trial. Psychooncology 2017;26:222-30.

27 Krusche A, Bradbury K, Corbett T, et al. Renewed: protocol for a randomised controlled trial of a digital intervention to support quality of life in cancer survivors. BMJ Open 2019;9:e024862.

28 Akechi T, Yamaguchi T, Uchida M, et al. Smartphone problem-solving and behavioural activation therapy to reduce fear of recurrence among patients with breast cancer (smartphone intervention to lessen fear of cancer recurrence: SMILE project): protocol for a randomised controlled trial. BMJ Open 2018;8:e024794.

29 Hong Y, Peña-Purcell NC, Ory MG. Outcomes of online support and resources for cancer survivors: a systematic literature review. Patient Educ Couns 2012;86:288-96.

30 Butow P, Smith Allan 'Ben', Smith A. Systematic reviews of pain and online interventions for cancer patients show evidence of mixed efficacy and highlight need for more rigorously designed research. Patient Educ Couns 2015;98:267-8.

31 Make it. Available: https://makeit-essen.medizin.uni-tuebingen.de [Accessed 08 Dec 2019].

32 Casellas-Grau A, Font A, Vives J. Positive psychology interventions in breast cancer. A systematic review. Psychooncology 2014;23:9-19.

33 Ringwald J, Marwedel L, Junne F, et al. Demands and needs for Psycho-Oncological eHealth interventions in women with cancer: cross-sectional study. JMIR Cancer 2017;3:e19.

34 Ringwald J, Gerstner L, Junne F, et al. [Mindfulness and Skills Based Distress Reduction in Oncology: The Web-Based PsychoOncological Make It Training]. Psychother Psychosom Med Psychol 2019;69:407-12.

35 Questback GmbH. Available: https://www.unipark.com [Accessed 08 December 2019]. 
36 Mehnert A, Müller D, Lehmann C, et al. Die Deutsche version des NCCN Distress-Thermometers Empirische Prüfung eines screening instruments Zur Erfassung psychosozialer Belastung bei Krebspatienten. Zeitschrift für Psychiatrie, Psychologie und Psychotherapie 2006;54:213-23.

37 Kroenke K, Strine TW, Spitzer RL, et al. The PHQ-8 as a measure of current depression in the general population. J Affect Disord 2009;114:163-73.

38 Spitzer RL, Kroenke K, Williams JBW, et al. A brief measure for assessing generalized anxiety disorder: the GAD-7. Arch Intern Med 2006;166:1092-7.

39 Schwarzer R, Jerusalem M. Skalen Zur Erfassung von Lehrer- und Schülermerkmalen. Dokumentation Der psychometrischen Verfahren Im Rahmen Der Wissenschaftlichen Begleitung des Modellversuchs Selbstwirksame Schulen. Berlin: Freie Universität Berlin, 1999.

40 Walach $\mathrm{H}$, Buchheld $\mathrm{N}$, Buttenmüller V, et al. Measuring mindfulness - the Freiburg mindfulness inventory (FMI). Pers Individ Dif 2006;40:1543-55.

41 Fliege H, Rose M, Arck P, et al. Validierung des "Perceived Stress Questionnaire" (PSQ) an einer deutschen Stichprobe. Diagnostica 2001;47:142-52.

42 Cella DF, Tulsky DS, Gray G, et al. The functional assessment of cancer therapy scale: development and validation of the general measure. J Clin Oncol 1993;11:570-9.

43 EuroQol Group. EuroQol--a new facility for the measurement of health-related quality of life. Health Policy 1990;16:199-208.
44 Brooke J. SUS: A ‘quick and dirty' usability scale. In: Jordan PW, Thomas B, Weerdmeester BA, et al, eds. Usability evaluation in industry. London: Taylor \& Francis, 1996: 189-94.

45 Boß L, Lehr D, Reis D, et al. Reliability and validity of assessing user satisfaction with web-based health interventions. J Med Internet Res 2016;18:e234.

46 Schröder J, Sautier L, Kriston L, et al. Development of a questionnaire measuring attitudes towards psychological online Interventions-the APOI. J Affect Disord 2015;187:136-41.

47 Christensen H, Griffiths KM, Farrer L. Adherence in Internet interventions for anxiety and depression. J Med Internet Res 2009;11:e13.

48 Waller R, Gilbody S. Barriers to the uptake of computerized cognitive behavioural therapy: a systematic review of the quantitative and qualitative evidence. Psychol Med 2009;39:705-12.

49 Palmqvist B, Carlbring P, Andersson G. Internet-Delivered treatments with or without therapist input: does the therapist factor have implications for efficacy and cost? Expert Rev Pharmacoecon Outcomes Res 2007;7:291-7.

50 Andersson G, Titov N, Dear BF, et al. Internet-Delivered psychological treatments: from innovation to implementation. World Psychiatry 2019;18:20-8.

51 Berger T, Hämmerli K, Gubser N, et al. Internet-Based treatment of depression: a randomized controlled trial comparing guided with unguided self-help. Cogn Behav Ther 2011;40:251-66. 